\title{
The Best Choice for Low and Moderate Myopia Patients Incapable for Corneal Refractive Surgery: Implantation of a Posterior Chamber Phakic Intraocular Lens
}

\section{Qin Wang}

Chongqing Aier Eye Hospital

\section{Lina Fan}

Chongqing Aier Eye Hospital

Qizhi Zhou ( $\triangle$ msagreon@126.com)

Chongqing Optometry Eye Hospital https://orcid.org/0000-0001-9392-4007

\section{Research Article}

Keywords: Low and moderate myopia, central hole phakic posterior chamber intraocular lens (ICL V4c) implantation, effectiveness, safety

Posted Date: October 25th, 2021

DOl: https://doi.org/10.21203/rs.3.rs-662075/v1

License: (c) (1) This work is licensed under a Creative Commons Attribution 4.0 International License.

Read Full License 


\section{The best choice for low and moderate myopia patients incapable for corneal refractive surgery: implantation of a posterior chamber phakic intraocular lens}

Qin Wang ${ }^{1}$, Lina Fan ${ }^{1}$, Qizhi Zhou*2

1 Chongqing Aier Eye Hospital, Chongqing 400020, China

*Corresponding author: Qizhi Zhou

Address: Aier Chongqing Optometry Eye hospital, No. 77, Daping Changiiang Second Road, Yuzhong, Chongqing 400040, China

Email: msagreon@126.com

Abstract

Purpose To assess the early clinical outcomes of central hole phakic posterior chamber intraocular lens(ICL V4c) implantation for correction low and moderate myopia, and to explore the relationship among arch height, intraocular pressure(IOP), and central anterior chamber depth(ACD).

Methods This retrospective clinical study included 27 patients (47 eyes )which underwent implantation of ICL V4c for correction of myopia with spherical equivalent between -1.75 D and -6.0 D, From January 2017 to December 2018. Preoperative corrected distance visual acuity (CDVA), postoperative uncorrected visual acuity (UCVA), and IOP were evaluated at one day, one week, three mouths, six mouths, and twelve months. ACD, arch height, and endothelial cell density (ECD) were evaluated at one, three and twelve months.

Results At 1 year follow-up, the UCVA of $75 \%$ eyes are larger than the preoperative CDVA, and that of $25 \%$ eyes are equal to the preoperative CDVA. The postoperative UCVA of the patients was higher than the preoperative BCVA and showed a stable trend. There was no significant difference in ECD $(\mathrm{P}>0.05)$. One month after surgery, the arch height was $(0.77 \pm 0.32) \mathrm{mm}$, and decreased to $(0.63 \pm 0.26) \mathrm{mm}$ at the one-year after surgery. ACD was $(3.24 \pm 0.25) \mathrm{mm}$ in the preoperative, decreased significantly to $(2.05 \pm 0.39) \mathrm{mm}$ at one month, and rose to $(2.2 \pm 0.39) \mathrm{mm}$ at one year after surgery. 
There was no significant correlation between IOP and ACD and arch height at 1 month and 1 year after operation. The correlation coefficient between ACD and arch height was -0.72 at 1 month after surgery, and it was -0.71 at 1 year. One patient had visual fatigue, one patient had glare, and none of subjects developed cataract, glaucoma and decompensation of corneal endothelium.

Conclusion ICL implantation is a safe, effective and stable method for the correction of low and moderate myopia, and it may be a good alternative for patients with low and moderate myopia who cannot undergo corneal refractive surgery.

keywords: low and moderate myopia; central hole phakic posterior chamber intraocular lens (ICL V4c) implantation; effectiveness; safety

\section{Introduction}

At present, the main surgical methods for correcting ametropia include corneal laser surgery and posterior chamber intraocular lens (ICL) implantation. Corneal laser surgery is effective and safe [1] for low and moderate myopia, but a contraindication for those with thin cornea or potential lesions such as suspicious keratoconus [2-4]. ICL may be a better choice for patients unsuitable for corneal laser surgery because it is reversible without corneal excision [5] and retains accommodation for eyes. Meanwhile it gives patients better visual acuity compared with laser-assisted in situ keratomileusis (LASIK) [6]. Besides, astigmatism posterior chamber intraocular lens (TICL) shows higher safety and gives patients better visual acuity in the treatment of moderate and high myopic astigmatism compared with PRK [7]. ICL currently becomes the first choice for patients with high myopia, and it is also favored by more and more people with low and moderate myopia. However, it also has some defects such as the formation of cataract, elevated IOP, TICL rotation, pigmented diffuse glaucoma, loss of endothelial cells, etc. [7] probably caused by the closely contacting with human lens. 
The most distinguishing feature of ICL V4c is that there is a central hole in the center of the lens with a diameter of about 360um, which can make aqueous humor flow directly into the anterior chamber through the pupil area, avoiding preoperative laser iris drilling and high intraocular pressure caused by pupil blockage. A large number of studies have reported its safety and effectiveness in correcting high myopia [8-9]. However, there are few reports on the correction of low and moderate myopia by ICL. In this work, a case-control study was conducted to explore the validity, safety and stability of ICL V4c implantation in the correction of low and moderate myopia.

\section{Subjects and methods}

This study was a retrospective review of the medical records of patients with low and moderate myopia who had ICL V4 implantation at Chongqing Aier General Ophthalmology Hospital from January 2017 to December 2018. The inclusion criteria were listed as follows: (1) the refraction remains stable for at least 1 year and the annual change was within $\pm 0.5 \mathrm{D}$; (2) the equivalent spherical lens (SE) was within -6.0 D; (3) the ACD $\geqslant 2.8 \mathrm{~mm}$; (4) the anterior chamber angle was open; (5) the ECD $>2,000$ cells $/ \mathrm{mm}^{2}$. Exclusion criteria were listed as follows: (1) patients younger than 18 years old, patients with Cataract, glaucoma, uveitis, retinal detachment, optic neuritis and other eye diseases; patients with diabetes, hypertension and other systemic diseases.

Preoperative examination included: UCVA and CDVA (measured in international standard visual acuity chart), manifest and cycloplegic refractions, IOP (KT-500, Kowa, Tokyo, Japan), endothelial cell density count(measured by Corneal endothelium microscope, NIDEK, Japan), corneal topography (Pentacam, OCULUS, Germany), central pachymetry (CT1000, Shin Nippon), anterior segment ultrasonic biomicroscopy (UBM) for sulcus to sulcus diameter, horizontal corneal diameter (white-to-white), ACD, and fundus examination (using slit lamp microscopy). All the implanted ICL V4c 
were produced by STAAR company in Switzerland. The diopter and model of ICL V4c were calculated by the lens calculation software (STAAR).

All ICL implantation surgeries were performed by a standardized method. One drop of levofloxacin ophthalmic (Towering pharmaceutical, China) was given three days before the operation, 4 times daily. Those who need to be implanted with astigmatic intraocular lens (TICL) were axially labeled before surgery. The patients were administered dilating and cycloplegic agents, and the surgery was performed under surface anesthesia with promecaine hydrochloride. All operations were performed by the same experienced surgeon. An auxiliary incision of $1 \mathrm{~mm}$ was created, and the anterior chamber was filled with viscoelastic material. Then, the main incision of $3 \mathrm{~mm}$ was created. The ICLV4c was pushed into the anterior chamber with an injector, adjusted to the center, and its loop was implanted into the ciliary sulcus. If it was astigmatic lens, the lens angle was marked according to the TICL surface line. Make it coincide with the preset corneal surface marker. At last, irrigation and aspiration were performed. After the surgery, the eye was coated with tobramycin Dexamethasone Eye Ointment (S.A. Alcon Couvreur N.V.), and covered with dressing.

The patients were followed up for 1 day, 1 week, 1 month, 3 months and 1 year. UCVA, CDVA, comprehensive optometry (before reaching the preoperative best corrected visual acuity), IOP, postoperative adverse reactions and complications were collected. ECD, ACD and arch height were collected in the postoperative examinations at 1 month and 1 year.

\section{Statistical analysis}

Statistical analysis was performed with SPSS22.0. Visual acuity data were converted to logarithm of the minimum angle of resolution (Log MAR) values for statistical analysis. Repeated measurement data were analyzed by repeated measurement variance. Paired 
data were compared by paired sample t-test, spearman or Pearson's method was used to study the correlation between IOP, ACD and arch height, and P value less than 0.05 was considered statistically significant.

\section{Results}

A total of 27 patients (47 eyes) with moderate and low myopia were enrolled in this study, aged from 19 to 37 years (27 males and 20 females), The mean spherical equivalent (SE) was $-4.55 \mathrm{D} \pm 1.05 \mathrm{D}$, and the mean preoperative spherical was $-4.28 \mathrm{D}$ $\pm 1.14 \mathrm{D}$, and the mean preoperative cylinder was - $0.54 \mathrm{D} \pm 0.67 \mathrm{D}$. There were no significant differences in patient preoperative demographic information.

Table 1 Comparison of preoperative CDVA (Log MAR) with postoperative UCVA (Log MAR) and preoperative and postoperative intraocular pressure $(x \pm s)$

\begin{tabular}{|c|c|c|c|c|c|c|}
\hline \multirow[b]{2}{*}{ Parameter } & \multirow{2}{*}{ Preoperative } & \multicolumn{4}{|c|}{ Postoperative } & \multirow[b]{2}{*}{$p$ value } \\
\hline & & $1 \mathrm{~d}$ & $1 \mathrm{wk}$ & $1 \mathrm{mo}$ & $1 \mathrm{y}$ & \\
\hline $\begin{array}{l}\text { Mean visual acuity } \\
(\log \text { MAR) }\end{array}$ & $0.01 \pm 0.04$ & $-0.01 \pm 0.05$ & $-0.06 \pm 0.06$ & $-0.06 \pm 0.06$ & $-0.05 \pm 0.06$ & $<0.01$ \\
\hline $\begin{array}{c}\text { Mean IOP } \\
(\mathrm{mm} \mathrm{Hg})\end{array}$ & $15.09 \pm 2.74$ & $13.54 \pm 3.19$ & $13.19 \pm 3.3$ & $12.31 \pm 2.89$ & $13.36 \pm 2.95$ & $<0.001$ \\
\hline
\end{tabular}

CDVA: corrected distance visual acuity; UDVA: uncorrected distance visual acuity; IOP: intraocular pressure; log MAR: logarithm of the minimum angle of resolution.

Table 1 shows the preoperative and postoperative visual acuity measurements. At the one-year postoperative visit, the postoperative UCVA of $75 \%$ of the eyes are larger than the preoperative CDVA, the others are equal to the preoperative CDVA, and the visual acuity at each review point was statistically significant $(\mathrm{F}=32.93, \mathrm{P}<0.01)$. In the pairwise comparison, there was no statistical significance $(\mathrm{P}>0.05)$ between UCVA ( $\log$ MAR) one day after surgery and CDVA ( $\log$ MAR) before surgery, and there was statistical significance $(\mathrm{P}<0.01)$ between $\mathrm{UCVA}$ other period after surgery $(\log$ MAR) and preoperative CDVA (log MAR). UCVA of patients after surgery was significantly improved compared with CDVA before surgery, and this trend was basically stable (Figure 1); 


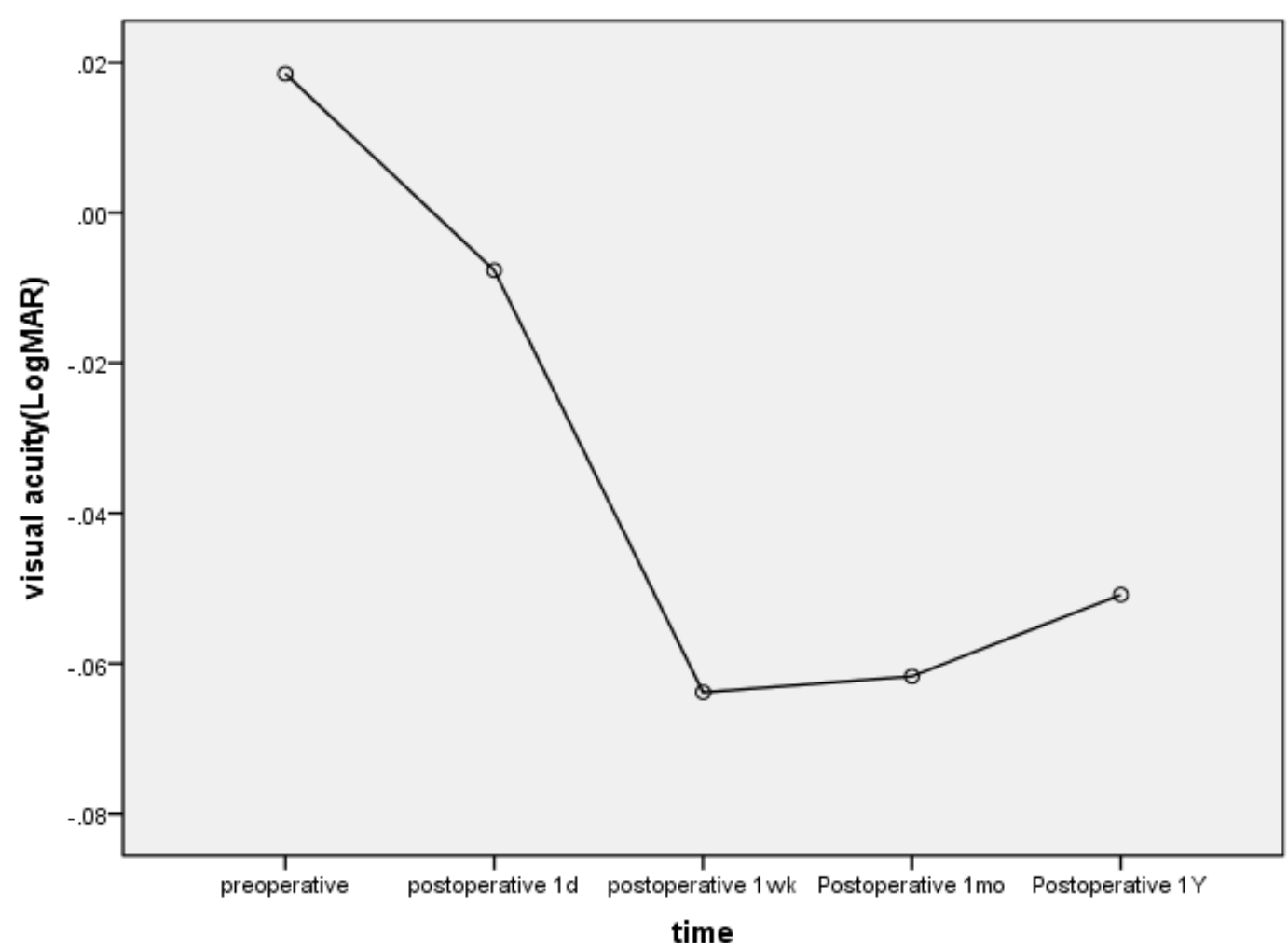

Figure 1. The change trend of visual acuity

There was significant difference between intraocular pressure before and after operation $(\mathrm{F}=32.7, \mathrm{P}<0.001)$. In pairwise comparison, there was no significant difference in IOP between 1 day and 1 year $(\mathrm{P}=1), 1$ month and 1 year after operation $(\mathrm{P}=0.098)$. The IOP was the lowest on the 1st week after operation, and tended to be stable after 1 month. The IOP decreased by $2.8 \mathrm{mmHg}$ at 1 month after operation, and $1.78 \mathrm{mmHg}$ at 1 year after operation, compared with that before operation (Figure 2). 


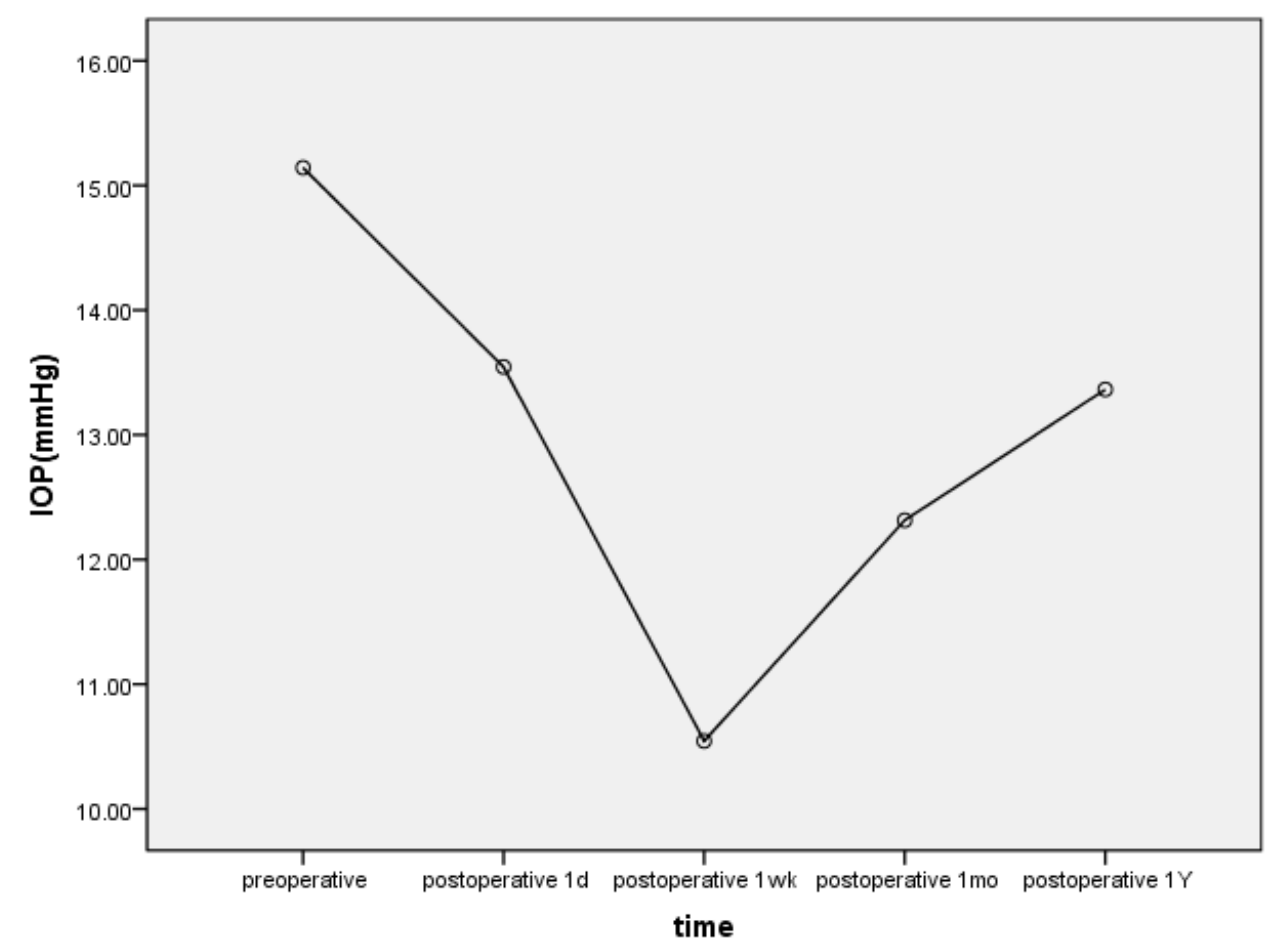

Figure 2 The change trend of intraocular pressure

There was no significant changes in the mean ECD at any timepoint $(\mathrm{F}=0.152$, $\mathrm{P}>0.05$ ), shown in Table 2. Table 2 also shows the central anterior chamber depth (ACD) before and at each time point after surgery. ACD decreased to the lowest in 1 month and increased in 1 year after surgery. At 1 month after surgery, the range of arch height was $0.22 \mathrm{~mm} \sim 1.42 \mathrm{~mm}$, the mean value was $0.77 \pm 0.32 \mathrm{~mm}$. It ranges from 0.17 $\mathrm{mm}$ to $1 \mathrm{~mm}$ at one-year after surgery, with an average of $0.63 \pm 0.26 \mathrm{~mm}$, and there was significant difference between arch height of the two timepoint $(\mathrm{t}=7.24, \mathrm{P}<0.05)$.

The correlation coefficient between arch height and ACD was $-0.72(\mathrm{P}<0.05)$ at 1 month after surgery and $-0.71(\mathrm{P}<0.05)$ at 1 year after surgery. ACD was negatively correlated with the change of arch height, and ACD deepened with the decrease of arch height.

There were no complications during the operation, one patient had visual fatigue and one patient had glare, and the symptoms were significantly relieved one month later. 
No complications such as cataract, glaucoma and corneal endothelial decompensation were found in all patients.

$\underline{\text { Table } 3 \text { Preoperative vs postoperative measurements }(\overline{\mathrm{x}} \pm \mathrm{s})}$

\begin{tabular}{|c|c|c|c|c|}
\hline \multirow{2}{*}{ Parameter } & \multirow{2}{*}{ Preoperative } & \multicolumn{2}{|c|}{ Postoperative } & \multirow{2}{*}{$p$ value } \\
\hline & & $1 \mathrm{mo}$ & $1 \mathrm{y}$ & \\
\hline ECD (cells/mm2) & $2915.45 \pm 286.92$ & $2906.03 \pm 309.1$ & $2915.49 \pm 334.77 *$ & 0.85 \\
\hline vault height (mm) & -- & $0.77 \pm 0.32$ & $0.63 \pm 0.26 \dagger$ & $<0.001$ \\
\hline $\operatorname{ACD}(\mathrm{mm})$ & $3.24 \pm 0.25$ & $2.05 \pm 0.39 *$ & $2.2 \pm 0.39 *$ & $\mathrm{P}<0.001$ \\
\hline
\end{tabular}

$\mathrm{ECD}=$ endothelial cell density; $\mathrm{ACD}=$ central anterior chamber depth

$* \mathrm{P}<0.05$ (compared with preoperative value), $\dagger \mathrm{P}<0.05$ (compared with 6-month postoperative value)

\section{Discussion}

ICL implantation can preserve corneal integrity, is not limited by corneal thickness, and has a wide range of correction of refractive errors. In recent years, ICL is no longer the second choice other than corneal laser surgery, but has become the first choice for more and more myopia patients. It is not only applicable for high myopia and ultra-high myopia patients, but also applicable for low and medium myopia patients with thin cornea and potential keratopathy. It is the irreplaceable method to correct myopia and its effectiveness and safety for the correction of low and moderate myopia had been reported [10]. Kamiya et al. [11] followed up 351 patients after ICL implantation, the results showed that ICL could correct not only high myopia, but also low to moderate myopia.

This study evaluated the clinical effect, safety and stability of implanting ICL in the correction of low to moderate myopia. Because the goal of ICL implantation is to obtain good visual acuity without glasses or contact lenses, we choose UDVA as one of the important clinical observation indexes. The results of this study show that ICL is an effective correction method for low and middle myopia patients, UDVA of 94\% eyes at one day after surgery was greater than or equal to CDVA before surgery, and the proportion reached $100 \%$ at one week after surgery. There was significant difference 
between postoperative and preoperative visual acuity (LogMAR) $(\mathrm{P}<0.01)$, but there was no significant difference between the visual acuity (LogMAR) at each recheck time point after surgery, which indicated that the visual acuity recovered quickly and tended to keep stable after implanting ICL. It is consistent with the previous study on the correction of middle and low myopia by ICL implantation [10].

The results of the study on intraocular pressure showed that there were significant differences in intraocular pressure at different time points $(\mathrm{P}<0.001)$. The intraocular pressure was $(13.54 \pm 3.19) \mathrm{mmHg},(10.54 \pm 2.9) \mathrm{mmHg},(12.31 \pm 2.89) \mathrm{mmHg}$ and (13.36 \pm 2.95$) \mathrm{mmHg}$, respectively. There was no statistical difference between 1 day and 1 year after operation $(\mathrm{P}=1)$, and there was no statistical difference between 1 month and 1 year $(\mathrm{P}=0.098)$. No patients had abnormal intraocular pressure. IOP is the lowest at one week after surgery. It is considered to be related to the use of IOPlowing drugs (acetazolamide tablets and bromonidine tartrate) after refractive surgery. At 2 3 weeks, the IOP is in a normal and stable state after the discontinuation of IOPlowing drugs. It is consistent with the previous studies on ICL implantation [5,12-13].

Elmohamady et al. [14] observed the changes of anterior chamber in 34 patients (34 eyes) with high myopia after implanting ICL. It was found that the chamber angle, anterior chamber volume and ACD decreased significantly in 1 month postoperatively, and there was no significant difference in 3 months, 6 months and 12 months postoperatively. The results of this study showed that the ACD was $(2.05 \pm 0.39) \mathrm{mm}$ at one month postoperatively and $(2.2 \pm 0.39) \mathrm{mm}$ at one year postoperatively, which was significantly decreased compared with that before the surgery $(3.24 \pm 0.25) \mathrm{mm}$. The main reason is the change of arch height. We found that the arch height was negatively correlated with ACD. The arch height at 1 month after surgery decreased by $18.18 \%$, compared with that at 1 year after surgery. 
Arch height is an important index to be monitored after implanting ICL. The optimum range of arch height is $0.25 \sim 0.75 \mathrm{~mm}$ [15]. A lower ICL vault after implantation is closely related to lens opacity [16]. High arch height can lead to shallow anterior chamber, angle closure, corneal endothelial damage, pupil occlusion or glaucoma [17]. In our study, the arch height ranges from $0.22 \mathrm{~mm}$ to $1.42 \mathrm{~mm}$ at one month after surgery, with an average value of $(0.77 \pm 0.32) \mathrm{mm}$, and it ranges from 0.17 $\mathrm{mm}$ to $1 \mathrm{~mm}$ at one year after surgery, with an average value of $(0.63 \pm 0.26) \mathrm{mm}$. The change of arch height presents a downward trend, consistent with Schmidinger [18]. In this study, arch height was negatively correlated with $\mathrm{ACD}$, with a correlation coefficient of 0.72 at 1 month after surgery and 0.71 at 1 year after surgery. At 1 month after surgery, $51.06 \%$ arch height are higher than $0.75 \mathrm{~mm}$ and $6.4 \%$ are less than $0.25 \mathrm{~mm}$. At 1 year after surgery, $23.4 \%$ arch height are higher than $0.75 \mathrm{~mm}$ and $6.4 \%$ are less than $0.25 \mathrm{~mm}$. It indicates that the change of arch height tends to a downward trend, but also tends to develop in the direction of safety.

The relevant research results of the US Food and Drug Administration showed that the loss rates of ECD at 1 year, 2 years and 3 years after surgery were $3 \%, 5.3 \%$ and $8.5 \%$, respectively. The loss rate of ECD in the early postoperative period was $9.9 \%$ (one month after the surgery), and then decreased to $4.7 \%$ (six months after the surgery). The number of ECD remained stable during the follow-up of 2 10 years [19-20]. In our study, the preoperative ECD count was $(2915.45 \pm 286.92) \mathrm{mm}^{2}$ and the postoperative ECD count was $(2906.03 \pm 309.1) \mathrm{mm}^{2}$ at one month, which was $0.3 \%$ lower than the preoperative ECD count. The postoperative ECD count was (2915.49 \pm 334.77$) \mathrm{mm}^{2}$ at 1 year, which was equal to that before surgery.

The visual acuity was significantly improved after ICLV4c implantation for the correction of moderate and low myopia, but glare in some patients is still a difficult 
problem to be solved, and it is also the current research hotspot and difficulty. In our study, even though one patient had binocular fatigue and one patient had glare, the symptoms were significantly relieved after one month, and no complications such as cataract, glaucoma and corneal endothelial decompensation were found in the other subjects.

\section{Conclusions}

Our study confirmed the effectiveness and stability of visual outcomes and safety after ICLv4c implantation in patients with middle and low degree myopia who can't undergo corneal refractive surgery. Arch height tends to decrease over time. But we need more cases and longer follow-up time to observe its effectiveness and safety, especially the changes of arch height and the development of anterior lens opacification, and to make theoretical preparation for the choice of ICL size in low to moderate myopia.

\section{Funding}

This work was supported by the Scientific Research Fund of Health Department of Hunan Province (2020SK50103).

\section{Conflicts of interest/competing interests}

The authors declare that they have no known competing financial interests or personal relationships that could have appeared to influence the work reported in this paper.

\section{Code availability}

N/A.

\section{Authors' contributions}

Qin Wang conceived the study, designed the study, collected data, performed the statistical analysis, interpreted data and drafted the manuscript. Qizhi Zhou collected 
data and supervised the study. Lina Fan conceived the study, collected data. All authors have carefully read, critically revised and approved the current version of the manuscript.

\section{Ethics approval}

All procedures performed in studies involving human participants were in accordance with the ethical standards of the institutional and/or national research committee and with the 1964 Helsinki declaration and its later amendments or comparable ethical standards. This study was approved by the Medical Ethics Committee of Chongqing Aier General Ophthalmology Hospital.

\section{Consent to participate}

All participants gave written informed consent for participation in the study.

\section{Consent for publication}

All participants gave written informed consent for their data to be published.

\section{Reference}

1.Tanzer DJ, Brunstetter T, Zeber R, Hofmeister E, Kaupp S, Kelly N, Mirzaoff M, Sray W, Brown M, Schallhorn S (2013) Laser in situ keratomileusis in United States Naval aviators. J Cataract Refract Surg 39:1047-1058.

2. Randleman JB, Russell B, Ward MA, Thompson KP, Stulting RD. (2003) Risk factors and prognosis for corneal ectasia after LASIK. Ophthalmology 110:267-275.

3. Binder PS, Lindstrom RL, Stulting RD, Donnenfeld E, Wu H, McDonnell P, Rabinowitz Y. (2005) Keratoconus and corneal ectasia after LASIK. J Refract Surg 21:749-752

4. Binder PS (2008) Risk factors for ectasia after LASIK. J Cataract Refractive Surg 34:20102011.

5. Packer M (2016) Meta-analysis and review: effectiveness, safety, and central port design of the intraocular collamer lens. Clin Ophthalmol 10:1059-1077. https://doi.org/10.2147/OPTH.S111620

6. Kazutaka Kamiya, Akihito Igarashi, Kimiya Shimizu, Kazuhiro Matsumura, Mari Komatsu 
(2012) Visual performance after posterior chamber phakic intraocular lens implantation and wave front-guided laser in situ keratomileusis for low to moderate myopia. Am J Ophthalmol $153: 1178-1186$.

7. Schallhorn S, Tanzer D, Sanders DR, Sanders ML(2007) Randomized prospective comparison of visian toric implantable collamer lens and conventional photorefractive keratectomy for moderate to high astigmatism. J Refract Surg 23:853-67.

8. Tian Y, Jiang HB, Jiang J, Wen D, Xia XB, Song WT(2017) Comparison of implantable Collamer lens Visian ICLV4 and ICLV4 for high myopia: A cohort study. Medicine (Baltimore) 96(25):e7294.

9. Hosny HM, Shalaby AM (2013) Visian implantable contact lens versus AcrySof Cachet phakic intraocular lenses: comparison of aberrmetric profiles. Clin Ophthalmol 7:1477-1486.

10. Dougherty PJ, Priver T (2017) Refractive outcomes and safety of the implantable collamer lens in young low-to-moderate myopes. Clin Ophthalmol 11:273-277.

11. Kamiya K, Shimizu K, Igarashi A, Kitazawa Y, Kojima T, Nakamura T, Oka Y, Matsumoto R (2018) Posterior chamber phakic intraocular lens implantation: comparative multicenter study in 351 eyes with low-to-moderate or high myopia. Br J Ophthalmol 102:177-181.

12. Ivo Guber, Victoria Mouvet, Ciara Bergin, Sylvie Perritaz, Philippe Othenin-Girard, François Majo (2016) Clinical outcomes and cataract formation rates in eyes 10 years after posterior phakic lens implantation for myopia. JAMA Ophthalmol 134:487-494.

13. Jae Hwan Choi, Dong Hui Lim, Seung Wan Nam, Chan Min Yang, Eui Sang Chung, TaeYoung Chung (2019) Ten-year clinical outcomes after implantation of a posterior chamber phakic intraocular lens for myopia. J Cataract Refract Surg 45:1555-1561.

14. Elmohamady MN, Abdelghaffar W (2017) Anterior Chamber Changes After Implantable Collamer Lens Implantation in High Myopia Using Pentacam: A Prospective Study. Ophthalmology and Therapy 6:343-349.

15. Alfonso JF, Fernández-Vega L, Lisa C, Fernandes P, González-Meijome J, Montés-Micó R (2012) Long-term evaluation of the central vault after phakic Collamer ${ }^{\circledR}$ lens (ICL) implantation using OCT. Graefe's Arch Clin Exp Ophthalmol 250:1807-1812

16. Lindland A, Heger H, Kugelberg M, Zetterström C(2010) Vaulting of myopic and toric Implantable Collamer Lenses during accommodation measured with Visante optical coherence 
tomography. Ophthalmology 117:1245-1250.

17. Arturo Gomez-Bastar, Martha Jaimes, Enrique O Graue-Hernández, Tito RamirezLuquin, Arturo Ramirez-Miranda, Alejandro Navas (2014) Long term refractive outcomes of posterior chamber phakic (spheric and toric implantable collamer lens) intraocular lens implantation. Int Ophthalmol 34:583-590.

18. Gerald Schmidinger, Birgit Lackner, Stefan Pieh, Christian Skorpik (2010) Long-term changes in posterior chamber phakic intraocular collamer 1ens vaulting in myopic patients. Ophthalmology 117:1506-1511.

19. Jiménez-Alfaro I, Benítez del Castillo JM, García-Feijoó J, Gil de Bernabé JG, Serrano de La Iglesia JM (2001) Safety of posterior chamber phakic intraocular lenses for the correction of high myopia: anterior segment changes after posterior chamber phakic intraocular lens implantation. Ophthalmology 108:90-99.

20. Paolo M Pesando, Maria Pia Ghiringhello, Giuseppe Di Meglio, Giordano Fanton Affiliations expand (2007) Posterior chamber phakic intraocular lens (ICL) for hyperopia: tenyear follow-up. J Cataract Refract Surg 33:1579-1584. 